\title{
The Pros and Cons of Helicobacter pylori Treatment: A Focus on Cardiovascular Mortality
}

\author{
Cheal Wung Huh and Byung-Wook Kim
}

Division of Gastroenterology, Department of Internal Medicine, Incheon St. Mary's Hospital, College of Medicine, The Catholic University of Korea, Seoul, Korea

See "Effect of Helicobacter pylori Treatment on Long-term Mortality in Patients with Hypertension" by Young-II Kim, et al. on page 47, Vol. 14, No. 1, 2020

The eradication of Helicobacter pylori has reduced the incidence of gastric cancer and mortality in asymptomatic individuals. ${ }^{1} H$. pylori eradication also significantly reduced metachronous gastric cancer risk in patients with early gastric cancer treated with endoscopic resection. ${ }^{2}$ Clarithromycin is the most commonly used $H$. pylori treatment regimen and has been reported to increase the risk of short-term cardiovascular mortality. ${ }^{3-5}$ In addition, the association between the use of clarithromycin and an increased risk of non-gastric cancer has been reported. ${ }^{2,6}$

However, cardiovascular disease and cancer are multifactorial diseases caused by multiple etiological factors. Therefore, the relationship between the use of clarithromycin and cardiovascular mortality or cancer development requires robust evidence to support. Recent several studies also showed inconsistent results regarding the association between clarithromycin use and overall mortality. ${ }^{3-5,7,8}$

In this issue of Gut and Liver, Kim et al. ${ }^{7}$ retrospectively analyzed 198,847 patients who treated for hypertension, based on data retrieved from the Korean National Health Insurance Service-National Sample Cohort database between 2002 and 2010. Patients in the $H$. pylori treatment cohort (5,541 patients) were matched with those in the nontreatment cohort (11,082 patients) at a ratio of 1:2. In the $H$. pylori treatment cohort, 5,342 patients (96.4\%) were prescribed with a triple therapy containing clarithromycin. During a median follow-up of 4.8 years, the overall mortality rate was significantly lower in the $H$. pylori treatment cohort compared with the nontreatment cohort (4.1\% vs 5.5\%). In terms of cause-specific mortality, when compared with the nontreatment cohort, the mortality risk due to cerebrovascular disease was significantly decreased in the $H$. pylori treatment cohort (adjusted hazard ratio, 0.46; 95\% confidence interval, 0.26 to $0.81 ; p=0.007)$. On the other hand, the mortality risk due to cardiovascular disease and cancer was not significantly different between the treatment and the nontreatment cohorts. Despite the absence of any difference in mortality rate, the risk for cardiovascular disease and overall cancer incidence were significantly higher in the $H$. pylori treatment cohort than in the nontreatment cohort. The authors speculated that the increased overall cancer incidence in the $H$. pylori treatment cohort might be related to differences in health behavior between the cohorts.

Until now, several cohort studies showed inconsistent results of association between clarithromycin-containing triple therapy and overall mortality. ${ }^{3-5,7,8}$ Recent cohort studies from the United Kingdom and Hong Kong found higher short-term cardiovascular risks with $H$. pylori treatment containing clarithromycin., However, a Danish cohort study of ischemic heart disease patients found similar overall and cardiovascular mortality after $H$. pylori treatment with or without clarithromycin. ${ }^{5}$ These studies included patients who had cardiovascular diseases at baseline. Meanwhile, this study excluded patients with cardiovascular disease other than hypertension at baseline. ${ }^{7}$ There was no association between clarithromycin-containing triple therapy and overall mortality in this study. This finding indicates that $H$. pylori treatment was not related to overall mortality, consistent with previous studies. ${ }^{1,2}$ To date, there is little evidence that $H$. pylori treatment increases the risk of mortality in patients without cardiovascular diseases.

\footnotetext{
Correspondence to: Byung-Wook Kim

Division of Gastroenterology, Department of Internal Medicine, Incheon St. Mary's Hospital, College of Medicine, The Catholic University of Korea, 56 Dongsu-ro, Bupyeong-gu, Incheon 21431, Korea

Tel: +82-32-280-5052, Fax: +82-32-280-5987, E-mail: gastro@catholic.ac.kr pISSN 1976-2283 eISSN 2005-1212 https://doi.org/10.5009/gnl19193

(a) This is an Open Access article distributed under the terms of the Creative Commons Attribution Non-Commercial License (http://creativecommons.org/licenses/by-nc/4.0) which permits unrestricted non-commercial use, distribution, and reproduction in any medium, provided the original work is properly cited.
} 
Interestingly, although the mortality risk due to cardiovascular disease was not different between the $H$. pylori treatment and nontreatment cohorts, the $H$. pylori treatment cohort had significantly increased risks of cardiovascular disease incidence in this study. Although recent data pertaining to acute cardiovascular risks of clarithromycin have been mixed, clarithromycin should be used prudently in patients with cardiovascular diseases other than hypertension. Furthermore, since clarithromycin resistance has been increasing, bismuth quadruple therapy or tailored therapy may be a better treatment option in several respects, especially for patients diagnosed with cardiovascular diseases.

This study, which was performed in a large cohort of patients with hypertension, has important clinical implication because authors found that $H$. pylori treatment in patients with hypertension was not associated with an increased risk of overall mortality including cardiovascular mortality. H. pylori eradication reduced gastric cancer incidence and mortality in asymptomatic individuals and reduced metachronous gastric cancer risk in patients with early gastric cancer who were treated with endoscopic resection or surgery. However, serious adverse events, which may be associated with $H$. pylori treatment, should not be overlooked. Although still disputed, $H$. pylori treatment containing clarithromycin requires caution in patients with cardiovascular diseases other than hypertension.

\section{CONFLICTS OF INTEREST}

No potential conflict of interest relevant to this article was reported.

\section{ORCID}

Cheal Wung Huh

https://orcid.org/0000-0001-7327-8503

\section{REFERENCES}

1. Ford AC, Forman D, Hunt RH, Yuan Y, Moayyedi P. Helicobacter pylori eradication therapy to prevent gastric cancer in healthy asymptomatic infected individuals: systematic review and metaanalysis of randomised controlled trials. BMJ 2014;348:g3174.

2. Choi IJ, Kook MC, Kim YI, et al. Helicobacter pylori therapy for the prevention of metachronous gastric cancer. N Engl J Med 2018;378:1085-1095.

3. Wong AY, Root A, Douglas IJ, et al. Cardiovascular outcomes associated with use of clarithromycin: population based study. BMJ 2016;352:h6926.

4. Root AA, Wong AY, Ghebremichael-Weldeselassie Y, et al. Evaluation of the risk of cardiovascular events with clarithromycin using both propensity score and self-controlled study designs. Br J Clin Pharmacol 2016;82:512-521.

5. Andersen SS, Hansen ML, Norgaard ML, et al. Clarithromycin use and risk of death in patients with ischemic heart disease. Cardiology 2010;116:89-97.

6. Boursi B, Mamtani R, Haynes K, Yang YX. Recurrent antibiotic exposure may promote cancer formation: another step in understanding the role of the human microbiota? Eur J Cancer 2015;51:2655-2664.

7. Kim YI, Kim YA, Lee JW, et al. Effect of Helicobacter pylori treatment on long-term mortality in patients with hypertension. Gut Liver 2020;14:47-56.

8. Mosholder AD, Lee JY, Zhou EH, et al. Long-term risk of acute myocardial infarction, stroke, and death with outpatient use of clarithromycin: a retrospective cohort study. Am J Epidemiol 2018;187:786-792. 